\title{
COMPARATIVE STUDY BETWEEN RADIAL BASIS FUNCTION NEURAL NETWORK AND RANDOM FOREST ALGORITHM FOR BUILDING ENERGY ESTIMATION
}

\author{
Che Munira Che Razali.*, Shamsul Faisal Mohd Hussein., Amrul Faruq., Shahrum \\ Shah Abdullah.
}

\begin{abstract}
Biologically Inspired System and Technology (Bio-Ist) iKohza, Department of Electronic Systems Engineering (ESE), Malaysia-Japan International Institute of Technology (MJIIT), Universiti Teknologi Malaysia Kuala Lumpur, MALAYSIA.

*munira2484@gmail.com

Tel: 013-7301744
\end{abstract}

\begin{abstract}
Recently, a computer experiment is ubiquitous in modeling and engineering design. Estimation of energy building efficiency using computer experiment is widely used to improve performance and energy consumption in the residential building. This paper proposed Radial Basis Function Neural Network (RBFNN) for energy building consumption dataset and make comparative studies between the Random Forest algorithm (RF) in previous work. This study using the experimental dataset in the literature that consists of 768 experimental data with eight input variables and two output parameters of estimation. The inputs variables are relative compactness, surface area, wall area, roof area, overall height, orientation, glazing area, and glazing area distribution of a building, while output variables include heating and cooling loads of the building. The analytical result of energy building performance shows RBFNN is better than RF algorithm in estimation based on error validation calculation using Mean Square Error (MSE), Mean Absolute Error (MAE) and Mean Relative Error (MRE). The findings of this comparative studies found that RBFNN is good in estimation based on accuracy performance, but the RF algorithm is suitable to determine irrelevant features in estimation by uses many decision trees simultaneously.
\end{abstract}

Keywords: Radial Basis Function Neural Network, Random Forest Algorithm, building Energy consumption, Estimation

\section{INTRODUCTION}

Malaysia has a good potential for energy resources such as oil, natural gas, coal, biomass, solar and hydro. Recently, energy consumption research surprisingly increases due to the human needs and energy sustainability. Building energy efficiency relied on heating load (HL) and cooling load (CL). The amount of energy needed to heat and cool a building depends on building envelope. Therefore, energy consumption of building needs to be optimized to keep HL and CL to a minimum[1]. In line with the growing interest in energy consumption, computer simulation is widely used in many applications especially in the engineering field. There are many statistical and machine learning method has been developed to estimate and predict HL and CL in building.

Artificial Neural Network (ANN) is the powerful technique for prediction and function approximation. ANN consist three layers which is input nodes, hidden layer, and output nodes. Previous study by W. Mai et al [2] shows RBFNN produced accurately forecasting model on an electric load of a commercial office building. Although there are many powerful and improved method of estimation and prediction in energy consumption, there is no empirical evidence that the algorithm is significantly and suitable for every dataset and case studies. A significant studies electricity prediction found that ANN performed marginally better than the random forest. However, RF can be used for handling high- dimensional data performs internal cross-validation (i.e., using ОOB (out-of-bag) samples) and only has a few tuning parameters[4]. 
This paper proposed comparative studies between random forest algorithm (RF) used in [5] and RBFNN for estimation building energy efficiency.

\section{METHODOLOGY}

Building energy dataset obtained from UCI Machine Learning is used to estimate building energy performance for HL and CL using RBFNN and RF. The dataset consists eight input variables that are: (1) relative compactness; (2) surface area; (3) wall area; (4) roof area; (5) overall height; (6) orientation; (7) glazing area and (8) glazing area distribution. The dataset was simulated using RBFNN and RF on a computer with the following specification: (1) Microsoft Windows 10 Professional Edition; (2) MATLAB R2017a; (3) Intel ${ }^{\circledR}$ Core $^{\mathrm{TM}}$ i7-6700K Processor. The result of RBFNN and RF that obtained from computer simulation compared relied on the mean absolute error (MEA), mean square error (MSE) and mean relative error (MRE). Furthermore, the effect of normalization, non-normalization, standardization, hold-out and cross-validation on the accuracy of estimation was analyzed and compared for both RF and RBFNN. In previous studies of normalization, Robinson et al. state that normalization aims to remove systematic technical effects that occur in the data to ensure that technical bias has minimal impact on the results[6].

\section{DISCUSSION AND CONCLUSION}

Further analysis of the result shows that comparison RBFNN produces better performance compare to the RF algorithm. Table 1 and Table 2 shows a comparison MEA, MSE, and MRE for HL and CL.

Table 1: Comparison MEA, MSE, and MRE performance for Heating Load

\begin{tabular}{|l|c|c|c|}
\hline \multirow{2}{*}{ Method } & \multicolumn{3}{|c|}{ Result } \\
\cline { 2 - 4 } & MEA & MSE & MRE \\
\hline RBFNN & 0.32 & 0.21 & 1.60 \\
\hline RF & 0.51 & 1.03 & 2.18 \\
\hline
\end{tabular}

Although RBFNN produces good performance than $\mathrm{RF}$, however, $\mathrm{RF}$ can determine variables importance input Based on the experiment result, ANN showed higher accuracy on a recently
Table 2: Comparison MEA, MSE, and MRE performance for Cooling Load

\begin{tabular}{|l|c|c|c|}
\hline \multirow{2}{*}{ Method } & \multicolumn{3}{|c|}{ Result } \\
\cline { 2 - 4 } & MEA & MSE & MRE \\
\hline RBFNN & 0.89 & 2.78 & 3.20 \\
\hline RF & 1.42 & 6.59 & 4.62 \\
\hline
\end{tabular}

acquired data. However, from results, it is concluded that both of the models are accepted for prediction and produced a good performance.

\section{ACKNOWLEDGMENT}

We would like to express our gratitude to the Malaysia-Japan International Institute of Technology (MJIIT), Universiti Teknologi Malaysia (UTM) for the financial support.

\section{REFERENCES}

[1] I.E. Agency, Transition to Sustainable Buildings. 2013. doi:10.1787/9789264202955-en.

[2] W. Mai, C.Y. Chung, T. Wu, H. Huang, G.S., C.Y. Chung, S., T. Wu. 2014. IEEE PES Gen. Meet. / Conf. Expo. 2014: 15.

[3] C. Deb, F. Zhang, J. Yang, S.E. Lee, K.W. Shah. 2017. Renew. Sustain. Energy Rev. 74: 902-924.

[4] M.W. Ahmad, M. Mourshed, Y. Rezgui. 2017. Energy Build. 147: 77-89.

[5] A. Tsanas, A. Xifara. 2012. Energy Build. 49: 560-567.

[6] M.D. Robinson, A. Oshlack. 2010. Genome Biol. 11. 